\title{
Commentary \\ Polarity determination in breast tissue: desmosomal adhesion, myoepithelial cells, and laminin 1
}

\author{
Mina J Bissell ${ }^{1}$ and David Bilder ${ }^{2}$ \\ ${ }^{1}$ Life Sciences Division, Lawrence Berkeley National Laboratory, Berkeley, California, USA \\ ${ }^{2}$ Department of Cell and Developmental Biology, University California Berkeley, Berkeley, California, USA \\ Corresponding author: Mina J Bissell (e-mail: mjbissell@lbl.gov)
}

Received: 21 October 2002 Revisions received: 16 January 2003 Accepted: 22 January 2003 Published: 5 February 2003

Breast Cancer Res 2003, 5:117-119 (DOI 10.1186/bcr579)

(c) 2003 BioMed Central Ltd (Print ISSN 1465-5411; Online ISSN 1465-542X)

\begin{abstract}
In all epithelial organs, apicobasal polarity determines functional integrity and contributes to the maintenance of tissue and organ specificity. In the breast, the functional unit is a polar double-layered tube consisting of luminal epithelial cells surrounded by myoepithelial cells and a basement membrane. It is far from clear how this double-layered structure is established and how polarity is maintained. Two recent papers have shed some light onto this intriguing problem in mammary gland biology. The results point to desmosomes and laminin 1 as having crucial roles. However, some questions remain.
\end{abstract}

Keywords: breast acini, desmosomes, epithelial positioning, luminal and myoepithelial cells, polarity, laminin 1

\section{Introduction}

Every cell in every tissue and organ in vivo is polar, in that it is organized asymmetrically. Even when tissues seem to lack morphological asymmetry, the cells within the tissues can still be polarized. Homeostasis itself is dependent on the maintenance of polarity. This is particularly true in the secretory epithelium, where without correct polarity, fluids would not be transported to the appropriate compartments. Yet we know little about what determines epithelial polarity in organs and how it is established and maintained in vivo.

In the past decade, the power of genetics in Drosophila and Caenorhabditis elegans has allowed the elucidation of some of the molecules and the molecular mechanisms involved in establishing polarity during embryonic development (reviewed in $[1,2]$ ). The task has been more difficult in mammals, in which tissue structure can be much more complex and genetic manipulations time-consuming and expensive. In the absence of suitable animal models, many studies on the establishment of polarity have been performed with cells in culture. Much useful information on junctional complexes, cell-cell and cell-extracellular matrix (ECM) interactions has been derived from these studies (reviewed in [3-7]). However, cultured cells, particularly epithelial cells, are known to lose their tissue-specific functions in culture (reviewed in [8]). Even in instances where apicobasal epithelial polarity is maintained on twodimensional substrata, the manifestation of polarity on plastic and in vivo is markedly different. The existing threedimensional culture models of epithelial cells partly fulfill a gap between these two extremes $[9,10]$.

\section{Three-dimensional models to study the interaction of epithelial and myoepithelial cells in the breast}

Organs are made of multiple cell types. One of the beststudied organs, the mammary gland, contains two epithelial cell types, called luminal epithelial and myoepithelial cells, that together form the acini, the functional unit of the gland. Nevertheless, studies in three-dimensional cultures using both rodent and human mammary luminal epithelial cells indicate that these cells can establish apicobasal polarity and cell junctions even in the absence of myoepithelial cells, if cells are cultivated in the presence of an exogenous basement membrane, Matrigel (a reconstituted basement membrane gel) [11-13]. Apicobasal polarity can also be established on a flexible collagen $1 \mathrm{gel}$; under 
these conditions, as cell-cell contact is increased, the cells can make and deposit their own basement membrane and become functionally differentiated [14]. Since there is neither Matrigel nor floating collagen gels in vivo, what then determines acinar polarity in the mammary gland, and what molecules and forces help to maintain it?

This commentary is focused on two recent papers $[15,16]$ that used similar human breast epithelial cells, but different approaches, to arrive at complementary conclusions about the requirements for the formation of the acini-like 'doublelayered tube'. Both papers took advantage of techniques allowing the isolation of pure populations of luminal epithelial and myoepithelial cells from human breast, using tissues obtained from reduction mammoplasties $[17,18]$.

Adhesion, mediated through either cell-cell or cell-ECM interactions, is clearly important in setting up epithelial polarity. But can one of these kinds of adhesion be excluded in favor of the other? Is there a hierarchy, or are both needed simultaneously? In a comprehensive and informative review, Yeaman and colleagues [3] discuss in detail the literature on polarized epithelial cells and postulate that polarity is established initially by extrinsic cues (namely cell-cell and cell-ECM adhesions) leading to asymmetry in the membranes at the site of the cue that is then transmitted throughout the rest of the cell. These 'symmetry-breaking' adhesive cues could involve any member of the cellular junction complexes. At the most apical part of the lateral membrane, these include cell-cell junctions such as tight junctions, adherens junctions and desmosomes (reviewed in [5]). Basally, hemidesmosomes that mediate cell-ECM interactions are the primary candidates [19]. In theory, any or all of these could be required for setting correct polarity, or, conversely, disruption of any one could lead to loss of polarity.

In an elegant study, Runswick and colleagues [15] cultured mouse mammary cell lines in Matrigel, and incubated purified human luminal epithelial and myoepithelial cells in rotary cultures in suspension. The latter form the double-layered tube in rotary cultures and resemble the mammary gland acini in vivo, although with a somewhat smaller acinus size. Using these systems, the authors showed that desmosomes were crucial in establishing polarized structures under both conditions. Immunofluorescence studies suggested that ECM components were not found in association with these double-layered tubes. However, the addition of specific peptides that block the adhesion of E-cadherin and desmocollins (desmosomal cadherins) resulted in disturbances in polarity, and the structures in Matrigel or the double-layered tubes in solution failed to organize. The authors concluded that the desmosomes are crucial for the formation of the doublelayered acini, and that the ECM molecules in general and
Using the same cell types but a different assay, Gudjonsson and colleagues [16] came to a different conclusion. Petersen and colleagues [12] had previously shown that luminal epithelial cells in three-dimensional Matrigel make organized, polar acini, but that the same cells grown in collagen I gels express different integrins on their basal surface [20] and do not have correct polarity [21]. Gudjonsson and colleagues [16] took advantage of these findings and used the behavior of human luminal cells in collagen I as a means of assaying the function of myoepithelial cells in lumen formation. They showed that the addition of myoepithelial cells to luminal cells in collagen I corrected the polarity of luminal cells, and that this function was dependent on the production of laminin 1 but was not reproduced by laminin 5 and laminin 10/11, two prominent laminins expressed in the breast. They also found that $75 \%$ of breast cancer-derived myoepithelial cells tested did not produce laminin 1 and were unable to reverse the polarity of epithelial cells in collagen I gels. These studies therefore concluded that an important requirement for the polarity and formation of the doublelayered tubes was the ability of myoepithelial cells to synthesize laminin 1.

Thus, these two studies identify essential and distinct determinants of mammary acini polarity. It would be interesting now for Runswick and colleagues to test myoepithelial cells that can not produce laminin 1 to see whether they can still form the double-layered tubes in the absence of this molecule when the desmosomes are intact. Similarly, Gudjonsson and colleagues [16] did not address the requirement for desmosomes, and they should now do so in their model system. However, it is likely that both laminin 1 and desmosomes would be necessary in vivo. It should be pointed out that the interpretation of experiments conducted by Gudjonsson and colleagues [16] with purified laminins ex vivo might also be complicated by the fact that laminins are never presented to cells in isolation in vivo; rather, these proteins are usually organized into a three-dimensional multi-protein polymerized structure. When studies are conducted ex vivo with purified laminins, results must be interpreted to include the possibility that only laminin-1 maintains the capacity to polymerize by itself, whereas laminin 5 and laminin 10/11 do not.

Another intriguing area to investigate is the inability of tumor myoepithelial cells to produce laminin 1, which seems to render them unable to signal for apicobasal polarity. Why are tumor myoepithelial cells unable to make laminin 1 ? Is this due to mutations, silencing, or post-transcriptional regulation? The evidence in Gudjonsson and colleagues [16] points to regulation at the level of mRNA. Evidence for links between a loss of epithelial polarity and tumor-like growth and invasiveness also exist in Drosophila mutants $[1,22]$. The laminin 1 -impaired human 
tumor cells can thus be used as functional 'mutants' in signaling studies.

These experiments have provided new insight into the contributions of cell-cell and cell-ECM interactions to the formation of acinar polarity in culture. The molecular mechanisms for correct cell-cell interactions and positioning remain to be determined both for cells in solution and in collagen gels. Future work will also need to address how these interactions at the cell surface are coupled to the intracellular pathways that actually execute polarity by trafficking proteins (including junctional adhesion receptors) to specific sites on the membrane. Evidence derived from invertebrate genetics can provide insight, yet invertebrates have distinctly different junctional structures [2], and Drosophila even seems to lack intermediate filaments. Moreover, evidence for cell-ECM interactions in invertebrate epithelial polarity is lacking [23]. The availability of RNA technologies to disrupt specific gene function, which can be applied to vertebrate cell cultures, might aid in distinguishing the functional requirement for signaling pathways that connect two different cell types as well as the relative contributions of E-cadherin, desmocollin, and integrin-based junctions to the polarization of mammary epithelia.

One important task now is to determine what molecules are involved in the formation and maintenance of these double-layered tubes in vivo so that more accurate and physiological models can be created in culture.

\section{Competing interests}

MJB is a co-author on the article from Gudjonsson and colleagues [16].

\section{References}

1. Bilder D: PDZ proteins and polarity: functions from the fly. Trends Genet 2001, 17:511-519.

2. Knust E: Regulation of epithelial cell shape and polarity by cell-cell adhesion. Mol Membr Biol 2002, 19:113-120.

3. Yeaman C, Grindstaff KK, Nelson WJ: New perspectives on mechanisms involved in generating epithelial cell polarity. Physiol Rev 1999, 79:73-98.

4. Knust E: Control of epithelial cell shape and polarity. Curr Opin Genet Dev 2000, 10:471-475.

5. Tsukita $S$, Furuse $M$, Itoh $M$ : Multifunctional strands in tight junctions. Nat Rev Mol Cell Biol 2001, 2:285-293.

6. O'Brien LE, Zegers MM, Mostov KE: Opinion. Building epithelial architecture: insights from three-dimensional culture models. Nat Rev Mol Cell Biol 2002, 3:531-537.

7. Nelson WJ, Yeaman C: Protein trafficking in the exocytic pathway of polarized epithelial cells. Trends Cell Biol 2001, $11: 483-486$

8. Bissell $\mathrm{MJ}$ : The differentiated state of normal and malignant cells or how to define a 'normal' cell in culture. Int Rev Cytol 1981, 70:27-100.

9. Walpita D, Hay E: Studying actin-dependent processes in tissue culture. Nat Rev Mol Cell Biol 2002, 3:137-141.

10. Jacks $T$, Weinberg RA: Taking the study of cancer cell survival to a new dimension. Cell 2002, 111:923-925.

11. Barcellos-Hoff MH, Aggeler J, Ram TG, Bissell MJ: Functional differentiation and alveolar morphogenesis of primary mammary cultures on reconstituted basement membrane. Development 1989, 105:223-235.
12. Petersen OW, Ronnov-Jessen L, Howlett AR, Bissell MJ: Interaction with basement membrane serves to rapidly distinguish growth and differentiation pattern of normal and malignant human breast epithelial cells. Proc Natl Acad Sci USA 1992, 89:9064-9068.

13. Bissell MJ, Weaver VM, Lelievre SA, Wang F, Petersen OW Schmeichel KL: Tissue structure, nuclear organization, and gene expression in normal and malignant breast. Cancer Res 1999, 59:1757s-1763s.

14. Streuli $\mathrm{CH}$, Bissell MJ: Expression of extracellular matrix components is regulated by substratum. J Cell Biol 1990, 110:1405-1415.

15. Runswick SK, O'Hare MJ, Jones L, Streuli CH, Garrod DR: Desmosomal adhesion regulates epithelial morphogenesis and cell positioning. Nat Cell Bio/ 2001, 3:823-830.

16. Gudjonsson T, Ronnov-Jessen L, Villadsen R, Rank F, Bissell MJ, Petersen OW: Normal and tumor-derived myoepithelial cells differ in their ability to interact with luminal breast epithelial cells for polarity and basement membrane deposition. J Cell Sci 2002, 115:39-50.

17. Page MJ, Amess B, Townsend RR, Parekh R, Herath A, Brusten L, Zvelebil MJ, Stein RC, Waterfield MD, Davies SC, O'Hare MJ: Proteomic definition of normal human luminal and myoepithelial breast cells purified from reduction mammoplasties. Proc Natl Acad Sci USA 1999, 96:12589-12594.

18. Pechoux C, Gudjonsson T, Ronnov-Jessen L, Bissell MJ, Petersen OW: Human mammary luminal epithelial cells contain progenitors to myoepithelial cells. Dev Biol 1999, 206:88-99.

19. Weaver V, Lelievre S, Lakins J, Chrenek M, Jones J, Giancotti F, Werb Z, Bissell M: $\beta 4$ integrin-dependent formation of polarized three-dimensional architecture confers resistance to apoptosis in normal and malignant mammary epithelium Cancer Cell 2002, 2:205-216.

20. Howlett AR, Bailey N, Damsky C, Petersen OW, Bissell MJ: Cellular growth and survival are mediated by beta 1 integrins in normal human breast epithelium but not in breast carcinoma. J Cell Sci 1995, 108:1945-1957.

21. Lelievre SA, Weaver VM, Nickerson JA, Larabell CA, Bhaumik A, Petersen OW, Bissell MJ: Tissue phenotype depends on reciprocal interactions between the extracellular matrix and the structural organization of the nucleus. Proc Natl Acad Sci USA 1998, 95:14711-14716.

22. Gateff E: Malignant neoplasms of genetic origin in Drosophila melanogaster. Science 1978, 200:1448-1459.

23. Brown $\mathrm{NH}$ : Cell-cell adhesion via the ECM: integrin genetics in fly and worm. Matrix Biol 2000, 19:191-201.

\section{Correspondence}

Mina J Bissell, Life Sciences Division, Lawrence Berkeley National Laboratory, Berkeley, California 94720, USA. E-mail: mjbissell@lbl.gov 